\title{
Screening mass in the massive Schwinger model at infinite temperature
}

\author{
Yeong-Chuan Kao and Yu-Li Lee* \\ Department of Physics, National Taiwan University, Taipei, Taiwan
}

(Received 11 December 1997; published 3 August 1998)

\begin{abstract}
The massive Schwinger model is in the Higgs phase only at infinite temperature. We calculate its screening mass to one-loop order and make comparisons with related studies. [S0556-2821(98)06916-1]

PACS number(s): $11.10 . \mathrm{Wx}$
\end{abstract}

A complete understanding of the low-energy behaviors of gauge theories is a most important objective in particle and condensed matter physics. In general, a gauge theory can be in one of the following phases in $3+1$ dimensions: the Coulomb phase, the confining phase, and the Higgs phase. Alas, to determine which phase a theory is in is very difficult. Only in some supersymmetric gauge theories can we achieve good or partial understanding. We know a lot better about (1 $+1)$-dimensional theories basically because these theories are simpler and there are more theoretical tools available. A famous example is the $(1+1)$-dimensional Schwinger model (spinor electrodynamics in $1+1$ dimensions) [1]. Schwinger showed that when the spinors are massless his theory is solvable and is in the Higgs phase in which the potential $V(R)$ between two static electric test charges separated by a large distance $R$ is of the form $V(R) \sim e^{-\mu R} / R, \mu$ being the screening mass. The solvability is essentially due to the one-loop exactness of the vacuum polarization $\pi_{\mu \nu}$ $=\left(p^{2} \delta_{\mu \nu}-p_{\mu} p_{\nu}\right) \pi\left(p^{2}\right)$. More than a decade later, Coleman, Jackiw, and Susskind [2], by using a bosonization technique, proved that the theory is in the confining phase in which a confining potential $\sim \sigma R$ exists if the spinors are massive. The reason for the difference between the massless and massive cases is that only the massless fermion loop would result in a pole in $\pi\left(p^{2}\right)$ which gives screening mass to the gauge bosons. Therefore, a tiny mass for fermions will change the theory dramatically. Other $(1+1)$-dimensional field theories thoroughly investigated include the Thirring model and the Gross-Neveu model. The lessons obtained from the study of such low-dimensional theories [3] are a great aid in our effort to understanding more realistic theories.

Once we have a grip on the phase structure of a theory, we should next study the transitions between phases induced by changes of physical parameters, in particular temperature. It has been shown by Fischler, Kogut, and Susskind [4] that the massive Schwinger model is always in the confining phase for any finite temperature, but the string tension vanishes at infinite temperature when the theory moves into the Higgs phase. Let us note here that, for the Schwinger model, the Higgs phase is called the superconducting phase and the confining phase called the insulating phase in the condensed matter parlance.

In this article, we shall calculate the screening (photon)

\footnotetext{
*Present address: Department of Physics, National Tsing Hua University, Hsinchu, Taiwan
}

mass in the massive Schwinger model at infinite temperature. The issue of screening mass, though academic, is worthy of study, as several interesting issues come together in the investigation. One is the chiral anomaly, the other is the decoupling of fermions at high temperature. As is well known, in the imaginary time formulation of finite temperature field theory [5], the propagator for a spinless boson in two dimensions is

$$
G_{\beta}\left(\omega_{n}, k_{1}\right)=\frac{1}{(2 n \pi / \beta)^{2}+k_{1}^{2}+m_{B}^{2}},
$$

and the propagator for the spin- $\frac{1}{2}$ fermion is

$$
S_{\beta}\left(\omega_{n}, k_{1}\right)=\frac{[(2 n+1) / \beta \pi] \gamma_{0}+k_{1} \gamma_{1}-m_{F}}{[(2 n+1) / \beta \pi]^{2}+k_{1}^{2}+m_{F}^{2}}
$$

where $\beta$ is the inverse temperature $1 / T$. The Matsubara frequency $\omega_{n}$ is $\pi / \beta$ times even (odd) integer for bosons (fermions). At high temperature where $\beta$ is small, we can observe from the above propagators that first, the mass terms are not important and second, the bosonic fluctuation modes for $n \neq 0$ are suppressed and the fermions are naively expected to be effectively decoupled. The Schwinger model is an interesting exception to this naive expectation. It is true that the fermion mass will become less important as temperature goes up and the massive Schwinger model will behave more similar to the massless case at infinite temperature. However, it is not true that the fermions are decoupled at infinite temperature, otherwise the Higgs phase will not appear. The point is that the one-loop diagram will lead to the chiral anomaly which is not affected by temperature as first shown by Dolan and Jackiw [6]. The fermionic quantum fluctuations cannot be neglected in the Schwinger model. We shall now calculate the one-loop diagram and demonstrate that both the massive and massless cases have the same screening mass at one-loop level at infinite temperature. While there is no higher order correction for the massless case [6], we have not been able to make definite statements about higher order corrections for the massive case yet. Further discussions on this matter are given in the last section.

The model is defined by the following action in twodimensional Euclidean space:

$$
S=\int_{0}^{\beta} d x_{0} \int d x_{1}\left[-\frac{1}{4} F_{\mu \nu} F_{\mu \nu}+\bar{\psi}(i \not \partial-e A-m) \psi\right],
$$


where the bosonic fields $A_{\mu}$ and the fermionic fields $\psi$ obey the periodic and antiperiodic boundary conditions in imaginary time, respectively. Note that the coupling parameter $e$ has the same dimension as that of mass and the theory is super-renormalizable.

The one-loop contribution to the vacuum polarization tensor is

$$
\pi_{\mu \nu}\left(p_{0}, p_{1}\right)=\frac{e^{2}}{\beta} \sum_{n} \int_{-\infty}^{\infty} \frac{d k_{1}}{2 \pi} \operatorname{tr}\left[\gamma_{\mu} S_{\beta}(p+k) \gamma_{\nu} S_{\beta}(k)\right],
$$

where $p_{0}$ is the Matsubara frequency. Because of gauge invariance, the polarization tensor can be written as

$$
\pi_{\mu \nu}\left(p_{0}, p_{1}\right)=\left(p^{2} \delta_{\mu \nu}-p_{\mu} p_{\nu}\right) \pi\left(p_{0}, p_{1}\right) .
$$

The fact that temperature does not affect the tensor structure of $\pi_{\mu \nu}$ is due to the absence of transverse direction in two dimensions. We separate $\pi\left(p_{0}, p_{1}\right)$ into the zero temperature part $\pi^{0}\left(p_{0}, p_{1}\right)$ and a temperature-dependent part $\delta \pi\left(p_{0}, p_{1}\right)$ :

$$
\pi\left(p_{0}, p_{1}\right)=\pi^{0}\left(p_{0}, p_{1}\right)+\delta \pi\left(p_{0}, p_{1}\right) .
$$

Straightforward calculations give the following expression for $\delta \pi\left(p_{0}, p_{1}\right)$ :

$$
\begin{aligned}
\delta \pi\left(p_{0}, p_{1}\right)= & 2 \frac{e^{2} m^{2}}{p_{0}^{2}-p_{1}^{2}} \int_{-\infty}^{\infty} \frac{d k_{1}}{2 \pi}\left\{\frac{n_{F}\left(E_{+}\right)}{E_{+}}\right. \\
& \times\left[\frac{1}{\left(E_{+}-p_{0}\right)^{2}-E_{-}^{2}}+\frac{1}{\left(E_{+}+p_{0}\right)^{2}-E_{-}^{2}}\right] \\
& +\frac{n_{F}\left(E_{-}\right)}{E_{-}}\left[\frac{1}{\left(E_{-}-p_{0}\right)^{2}-E_{+}^{2}}\right. \\
& \left.\left.+\frac{1}{\left(E_{-}+p_{0}\right)^{2}-E_{+}^{2}}\right]\right\},
\end{aligned}
$$

where $E_{ \pm} \equiv \sqrt{\left(k_{1} \pm p_{1} / 2\right)^{2}+m^{2}}$ and $n_{F}(x) \equiv 1 /(\exp x / T+1)$. The answer given in the following is actually for the retarded (instead of the time-ordered) Green's function because the dispersion relation, which is checked to ascertain the correctness of the result, is most transparent in this form. The real and imaginary parts of $\delta \pi_{R}$ are

$$
\begin{aligned}
\operatorname{Re}\left\{\delta \pi_{R}(\omega, p)\right\}= & 2 \frac{e^{2} m^{2}}{\omega^{2}-p^{2}} \int_{-\infty}^{\infty} \frac{d k_{1}}{2 \pi}\left\{\frac{n_{F}\left(E_{+}\right)}{E_{+}}\right. \\
& \times\left[\frac{1}{\left(E_{+}-\omega\right)^{2}-E_{-}^{2}}+\frac{1}{\left(E_{+}+\omega\right)^{2}-E_{-}^{2}}\right] \\
& +\frac{n_{F}\left(E_{-}\right)}{E_{-}}\left[\frac{1}{\left(E_{-}-\omega\right)^{2}-E_{+}^{2}}\right. \\
& \left.\left.+\frac{1}{\left(E_{-}+\omega\right)^{2}-E_{+}^{2}}\right]\right\},
\end{aligned}
$$

$$
\begin{aligned}
& \operatorname{Im}\left\{\delta \pi_{R}(\omega, p)\right\} \\
&=-\frac{1}{2} \frac{e^{2} m^{2}}{\omega^{2}-p^{2}} \int_{-\infty}^{\infty} d k_{1} \\
& \times\left\{\frac { n _ { F } ( E _ { + } ) } { E _ { + } E _ { - } } \left[\delta\left(\omega-E_{+}-E_{-}\right)-\delta\left(\omega-E_{+}+E_{-}\right)\right.\right. \\
&\left.+\delta\left(\omega+E_{+}-E_{-}\right)-\delta\left(\omega+E_{+}+E_{-}\right)\right] \\
&+\frac{n_{F}\left(E_{-}\right)}{E_{+} E_{-}}\left[\delta\left(\omega-E_{-}-E_{+}\right)-\delta\left(\omega-E_{-}+E_{+}\right)\right. \\
&\left.\left.+\delta\left(\omega+E_{-}-E_{+}\right)-\delta\left(\omega+E_{-}+E_{+}\right)\right]\right\} .
\end{aligned}
$$

Though the complete form of the real part is not obtained, it can be checked after some calculations that

$$
\operatorname{Re}\left\{\delta \pi_{R}\left(\omega, q^{2}\right)\right\} \stackrel{T \rightarrow \infty}{\longrightarrow}-\operatorname{Re}\left\{\pi_{R}^{0}\right\}-\frac{e^{2}}{\pi} \frac{1}{q^{2}},
$$

with $q^{2} \equiv \omega^{2}-p^{2}$. Therefore,

$$
\operatorname{Re}\left\{\pi_{R}\left(\omega, q^{2}\right)\right\} \stackrel{T \rightarrow \infty}{\longrightarrow}-\frac{e^{2}}{\pi} \frac{1}{q^{2}} .
$$

If we calculate $\operatorname{Re}\left\{\pi_{R}\left(\omega, q^{2}\right)\right\}$ in the massless Schwinger model, we shall find that it is temperature independent and is exactly the same as the right-hand side of Eq. (11) [6]. This is related to the fact alluded to above that the chiral anomaly is not affected by temperature. The integral for the imaginary part of $\delta \pi_{R}$ can be computed exactly to be

$$
\begin{aligned}
\operatorname{Im}\left\{\delta \pi_{R}\left(\omega, q^{2}\right)\right\}= & -[\theta(\omega)-\theta(-\omega)] e^{2} \theta\left(1-4 m^{2} / q^{2}\right) \\
& \times \frac{2 m^{2}}{\left(q^{2}\right)^{2} \sqrt{1-4 m^{2} / q^{2}}}\left\{\theta\left(q^{2}\right)\right. \\
& \times\left[n_{F}\left(\frac{\omega}{2}-\frac{|p|}{2} \sqrt{1-4 m^{2} / q^{2}}\right)\right. \\
& \left.+n_{F}\left(\frac{\omega}{2}+\frac{|p|}{2} \sqrt{1-4 m^{2} / q^{2}}\right)\right]+\theta\left(-q^{2}\right) \\
& \times\left[n_{F}\left(\frac{\omega}{2}+\frac{|p|}{2} \sqrt{1-4 m^{2} / q^{2}}\right)\right. \\
& \left.\left.-n_{F}\left(-\frac{\omega}{2}+\frac{|p|}{2} \sqrt{1-4 m^{2} / q^{2}}\right)\right]\right\} . \quad \text { (12) }
\end{aligned}
$$

Together with the imaginary part of $\pi_{R}^{0}$,

$$
\begin{aligned}
\operatorname{Im}\left\{\pi_{R}^{0}\left(\omega, q^{2}\right)\right\}= & {[\theta(\omega)-\theta(-\omega)] } \\
& \times e^{2} \frac{2 m^{2}}{\left(q^{2}\right)^{2} \sqrt{1-4 m^{2} / q^{2}}} \theta\left(q^{2}-4 m^{2}\right),
\end{aligned}
$$

we find that 


$$
\begin{aligned}
\operatorname{Im}\left\{\pi_{R}\left(\omega, q^{2}\right)\right\} & \\
= & \frac{2 e^{2} m^{2}}{\left(q^{2}\right)^{2} \sqrt{1-4 m^{2} / q^{2}}} \theta\left(1-4 m^{2} / q^{2}\right) \\
& \times \frac{\sinh (\beta \omega / 2)}{\cosh (\beta \omega / 2)+\cosh (\beta|p| / 2) \sqrt{1-4 m^{2} / q^{2}}} .
\end{aligned}
$$

We shall check the above result against the following dispersion relation in the infinite temperature limit:

$$
\operatorname{Re}\left\{\pi_{R}\left(\omega, q^{2}\right)\right\}=\frac{1}{\pi} \int_{-\infty}^{\infty} d \omega^{\prime} \frac{\operatorname{Im} \pi_{R}\left(\omega^{\prime}, \omega^{\prime 2}-p^{2}\right)}{\omega^{\prime}-\omega} .
$$

Simple manipulation gives

$$
\int_{-\infty}^{\infty} d S \operatorname{Im}\left\{\pi_{R}(\omega, S)\right\}=e^{2} f(\alpha, \gamma)[\theta(\omega)-\theta(-\omega)],
$$

where

$$
\begin{aligned}
f(\alpha, \gamma) \equiv & \frac{1}{2} \int_{-\infty}^{\infty} d S \frac{1}{S^{2} \sqrt{1-1 / S}} \theta(1-1 / S) \\
& \times \frac{\sinh \alpha \gamma}{\cosh \alpha \gamma+\cosh \alpha \sqrt{\gamma^{2}-S} \sqrt{1-1 / S}},
\end{aligned}
$$

where $\alpha \equiv \beta m$ and $\gamma \equiv|\omega| / 2 m$. The fact that $f(0, \gamma)=1$, shows that, as $T \rightarrow \infty$,

$$
\operatorname{Im}\left\{\pi_{R}\left(\omega, q^{2}\right)\right\}=e^{2} \delta\left(q^{2}\right)[\theta(\omega)-\theta(-\omega)] .
$$

Equations (11) and (18) together verify that the dispersion relation [Eq. (15)] is obeyed at infinite temperature. The above discussion is in the spirit of Dolgov and Zakharov who originated the idea of using the dispersion relation to understand anomaly [7]. We find from Eq. (11) that the oneloop contribution to the squared screening mass $\mu^{2}$ at infinite temperature is $e^{2} / \pi \equiv \mu_{0}^{2}$, which is also the result in the massless Schwinger model.

There are two related studies in the literature. One is by Baier and Pilon [8] where they also obtained the same imaginary part of the polarization tensor. (Actually, they calculated the causal Green function using the real-time formalism.) They proved the temperature independence of the chiral anomaly in the limit of vanishing fermion mass. However, they did not discuss the infinite temperature limit when the fermion mass is kept finite so that the issue of screening mass was not investigated. The other more relevant study by Hosotani and Rodriguez [9] employed a nonperturbative approach to calculate the spectrum of the massive Schwinger model. They found a boson having mass $\sqrt{e^{2} / \pi+8 m^{2}}$ at infinite temperature. This result cannot be easily connected to any perturbative calculation. It should be noticed that this mass $\left(\sqrt{e^{2} / \pi+8 m^{2}}\right)$ can be smoothly connected to the screening mass $\mu_{0}$ in the massless Schwinger model as the fermion mass $m \rightarrow 0$. If a smooth connection between $\mu$ and $\mu_{0}$ holds true for the perturbative series term by term, then there is no higher order contributions to $\mu$ at infinite temperature. This is because the $(n+1)$-loop contribution to $\mu^{2} / \mu_{0}^{2}$ takes the form $C_{n}\left(e^{2} / m^{2}\right)^{n}$ at $T=\infty$, where $C_{n}$ is a dimensionless constant. If we make the not entirely unreasonable conjecture that $\mu^{2}$ is smoothly connected to $\mu_{0}^{2}$ order by order, then each $C_{n}, n>0$, can only be zero. That Hosotani and Rodriguez did not find a boson with mass $\mu_{0}$ at infinite temperature indicates that the conjecture is wrong, and the infinite temperature limit can only be taken after the summation of the perturbative series. Further work remains to be done to achieve a better understanding of the relationship between Hosotani and Rodriguez's result and ours.

This work was supported by the National Science Council of R.O.C. under Grant No. NSC84-2112-M-002-008.
[1] J. Schwinger, Phys. Rev. 128, 2425 (1962).

[2] S. Coleman, R. Jackiw, and L. Susskind, Ann. Phys. (N.Y.) 93, 267 (1975).

[3] E. Abdalla, M. C. B. Abdalla, and K. D. Rothe, Two Dimensional Quantum Field Theory (World Scientific, Singapore, 1991).

[4] W. Fischler, J. Kogut, and L. Susskind, Phys. Rev. D 19, 1188 (1979).
[5] J. Kapusta, Finite Temperature Field Theory (Cambridge University Press, Cambridge, England, 1989).

[6] L. Dolan and R. Jackiw, Phys. Rev. D 9, 3320 (1974).

[7] A. D. Dolgov and V. I. Zakharov, Nucl. Phys. B27, 525 (1971).

[8] R. Baier and E. Pilon, Z. Phys. C 52, 339 (1991).

[9] Y. Hosotani and R. Rodriguez, Phys. Lett. B 389, 1211 (1996). 